\title{
Hybrid FWF Model for Gaussian Noise Reduction from Images
}

\author{
Lubna Farhi*1, Farhan Ur Rehman ${ }^{2}$ \\ ${ }^{1}$ Sir Syed University of Engineering \& Technology \\ ${ }^{2}$ University of Toronto, Canada \\ Ilubnafarhi@yahoo.com,lfarhi@ssuet.edu.pk; ${ }^{2}$ fahan.rehman@mail.utoronto.ca
}

\begin{abstract}
In this paper, the image efficiency is improved by using hybrid model of wiener's filter and fuzzy filter. It's a challenging task to remove Gaussian noise (GN) from an image and to protect the picture edges. The Fuzzy - Wiener filter (FWF) hybrid model is used for optimizing the image smoothness and efficiency at a high level of GN. The efficiency is measured by using Structural Similarity (SSIM), Mean Square Error (MSE), and Peak Signal to Noise Ratio (PSNR). The proposed algorithm substitutes a mean value of the matrix for a non-overlapping block and replaces the total pixel number with each direction. In the proposed model, overall results presented that the optimized hybrid model FWF has an enormous computational speed and impulsive noise reduction, which enables efficient filtering as compared to the existing techniques
\end{abstract}

Keywords- Gaussian Noise, Weiner Filter, Fuzzy Filter, Noise Removal, Filtering Techniques

\section{INTRODUCTION}

The image de-noising is an essential part processing. In this digitalized world, the usage of imaginings in every field is becoming increasingly popular. As for as quality of image is concern the noise always present in digital images. It is basically unwanted information or unwanted data which makes the images blur and lower the quality. There are various types of noises present in digital images which can arise during the image acquisition, transmission, coding and processing steps [15]. The fundamental goal is to restore the original image by suppressing noise. Since filtering plays a vital role preprocessing of images, various types of filters have been applied to remove the noise in an image. The filtering approach has been proved to be the best when the image is corrupted with any of the statistical models we applied on the original image. Mathematical representation of the filtering technique as follows [1]:

\footnotetext{
Lubna Farhi*I, Farhan Ur Rehman ${ }^{2}$

${ }^{I}$ Sir Syed University of Engineering \& Technology

${ }^{2}$ University of Toronto, Canada

Ilubnafarhi@yahoo.com,lfarhi@ssuet.edu.pk;

fahan.rehman@mail.utoronto.ca
}

$$
\begin{aligned}
& G(x, y)=F(x, y) * U(x, y)+N(x, y) \\
& H(x, y)=R[G(x, y)]
\end{aligned}
$$

Where $F(x, y)$ is the acquired image, $U(x, y)$ is the degradation function, * represents convolution, $N(x, y)$ shows noise, $G(x, y)$ is the initial corrupted output image and $H(x, y)$ is the final filtered output [2]. The random noise is added in an image which can be modeled as Gaussian or normal distribution. The probability density function (PDF) which is almost equal to the normal distribution in a statistical noise model, known as Gaussian Noise [3].

Several filtration techniques have been investigated in the literature to reduce or remove the Gaussian noise. The most common filters are Weiner filter and Fuzzy filter. The Weiner filter is one of the most common filter in order to remove the Gaussian noise and it is usually applied in the signal processing and image processing community $[4,13]$. The existing Weiner filter is effective to reduce the noise but sometimes it produces blurring at the edges of the image [5] because it is spatially invariant. A Weiner filtering Kernel approach is utilized for removing the Gaussian noise from images [6]. The disadvantages of this technique is that in weight function, it assigned the weight value to the pixels. Hence, the made Weiner filter fails to retain the edges of the image and the some of the information is lost. The switching Weiner filter is used for image denoising in [7]. This method is only efficient for the impulse noise removal, not for continuous noise. The issues lie with weighting of pixels which may cause the loss of the entire image details. Fuzzy based filtering technique can reduce noise in a good way with supervised data or knowledge and is suitable for some applications in complicated scene analysis and object recognition [8, 14]. The researcher develops fuzzy rule based system for denoising the corrupted image. This system employs fuzzy rules for smoothing, sharpening and specially the edge detection [9].

To mitigate the shortcomings, a fuzzy-Weiner filter (FWF) hybrid model is proposed for removing the Gaussian noise from the images. In proposed model, the modified Weiner filter is proposed by inserting the parameters of fuzzy filter. The fuzzy filter works on all pixel directions (East, West, South, and North). And these pixel location ease to find the every pixel at the 
edge. The analysis shows that the proposed algorithm provides the clear image as compared to the existing filtering techniques.

\section{PROPOSED METHODOLOGY}

This paper investigates the enhanced filtering method by combining the existing Weiner and fuzzy filter technique. And then compared with each other using the performance metrics which are SSIM, MSE and PSNR. Wiener filter has noise reduction kernel with spatial domains and utilized to eliminate the noise from images. For enhancing the image quality, filter follows the following steps: firstly, select the size of matrix $(n X m)$ for the noise reduction filter. Secondly, the selected matrix is used to recompute the value of the new pixel compared to the degraded pixel value [10]. The Wiener filter involves both the variance and average pixel values in the $(n X m)$ matrix and mathematically can be represented as:

$\mu=\frac{1}{N \times M} \sum_{n, m € p} y(n, m)$

$\sigma=\sqrt{\frac{1}{N \times M} \sum_{n, m € p} y^{2}(n, m)}-\mu^{2}$

Where, mean $=\mu$, variance of noise $=\sigma,(n X m)$ is the size of the area $\mathrm{p}$, and $y(n, m)$ represents total number of pixels. The estimated values of the wiener filter are represented as:

$B_{w}(n, m)=\mu+\frac{\sigma^{2}-v^{2}}{\sigma^{2}} *[y(n, m)-\mu]$

Equation 5 gives the blurry image and edges are not preserved. Hence, the goal of this methodology is to increase image quality by inserting the fuzzy perimeters into the existing Weiner filter. The FWF technique is used to diminish the noise in images. In addition, this technique preserves the edges at the highest level of Gaussian noise (HLGN). The modified algorithm, which is based on the fuzzy filter, replaces the mean value by $n X m$ window size for non-overlapping blocking and the total number of pixel is replaced by every direction. The FMF is represented as follows:

$B_{F M W F}(\mathrm{n}, \mathrm{m})=\frac{f(x, y)}{n \times m}+\frac{\sigma^{2}-v^{2}}{\sigma^{2}} *\left(D-\frac{f(x, y)}{n \times m}\right)$

Where

$\mathrm{D}=[\mathrm{dN}$ (North), dS (South) dE (East) dW (West) ] ; $d N(n, m)=y(n-1, m)-y(n, m)$; $d S(n, m)=y(n+1, m)-y(n, m)$; $d E(n, m)=y(n, m+1)-y(n, m)$; $d W(n, m)=y(n, m-1)-y(n, m) ;$
This paper is organized as follows. Section 2 explains the proposed methodology. Experimental analysis have been done in section 3 . Section 4 describes the simulated result. Section 5 concludes this paper.

In this paper, performance metrics i.e. SSIM, MSE and PSNR are used to compare the performance of original image $[I(m, n)]$ and the filtered image $[K(m, n)]$.

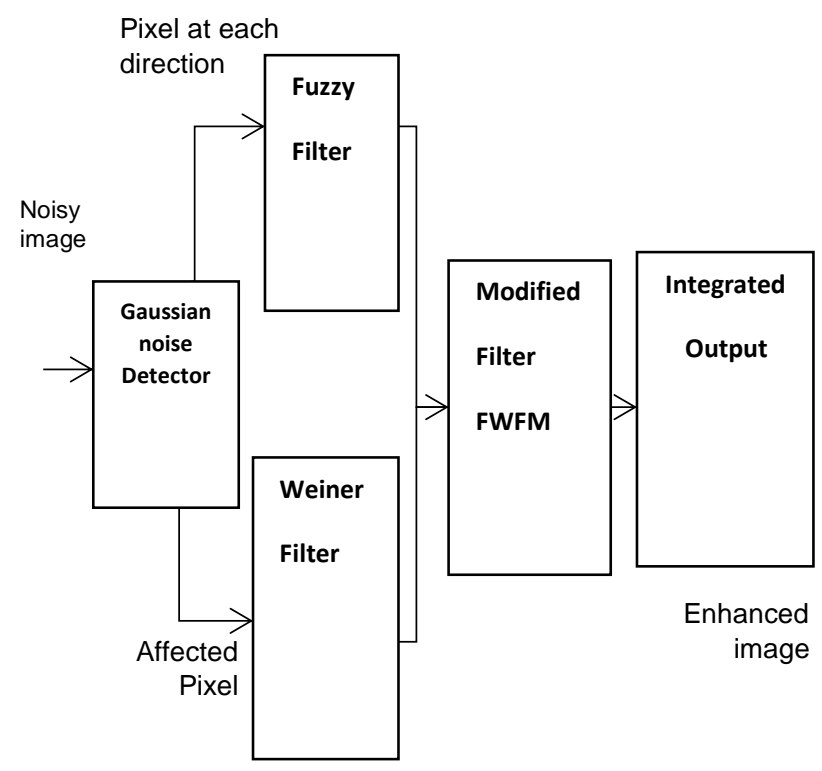

Fig. 1: Proposed System Model

The SSIM is the performance metric for quantifies the image quality degradation [12]. It is a method used to gauge the similarity between two images. The SSIM values are between 0 and 1 considered as a good quality image. Here, $\mathrm{L}$ is the range which is dynamic i.e. (2bits/pixels -1), $\mathrm{K} 1$ and $\mathrm{K} 2$ are constants i.e. 0.01 and 0.03 respectively and $\mathrm{C}_{1}=(\mathrm{K} 1 \mathrm{~L})^{2}, \mathrm{C}_{2}=(\mathrm{K} 2 \mathrm{~L})^{2}$ The calculated formula is :

$\operatorname{SSIM}(x, y)=\frac{\left(2 \mu_{x} \mu_{y}+C_{1}\right)\left(2 \sigma_{x y}+C_{2}\right)}{\left(2 \mu_{x}^{2} \mu_{y}^{2}+C_{1}\right)\left(\sigma_{x}^{2}+\sigma_{y}^{2}+C_{1}\right)}$

The MSE is the differences between the original image and the filtered image. The low value of MSE indicated the lower error in the image [11] which gives the good quality of image. Here $I(n, m)$ is the original image and $K(n, m)$ is filtered image. The calculated formula is given below:
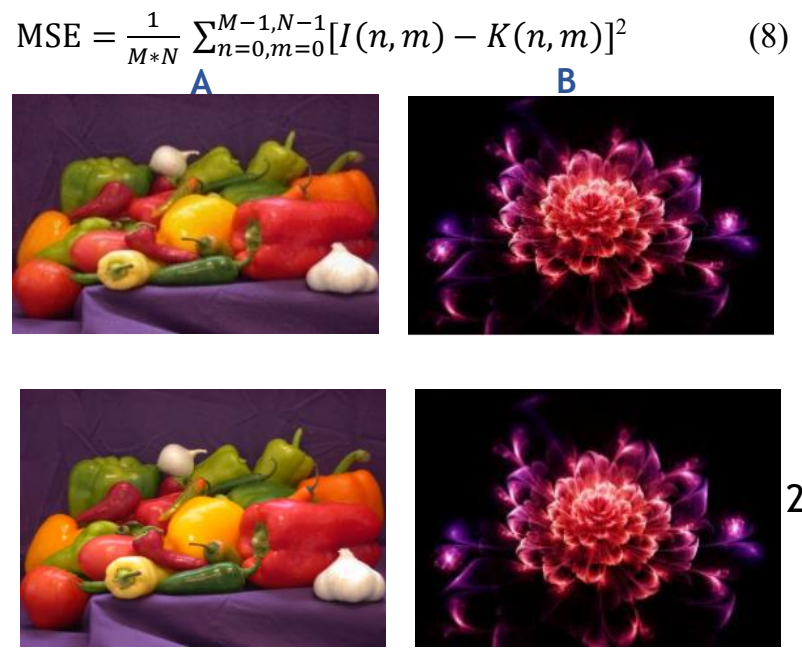
The ratio between the maximum power and the noise corrupting image is PSNR [12]. It also defined via the Root Mean Square Error (RMSE). High value of the PSNR gives the high quality of image. The PSNR is define as in Equation 9:

PSNR $=20 \log 10\left(\frac{255}{R M S E}\right)$

Where, $\quad$ RMSE $=\sqrt{\text { MSE }}$

\section{EXPERIMENTAL ANALYSIS}

This section of paper briefly discussed the experimental result analysis and also provides the detailed explanation of modified Weiner filter and their performance metrics. Comparative and quantitative analysis of fuzzy modified Weiner filter (FMWF) has been evaluated.

\subsection{Experimental Setup}

The FWF hybrid model was experimented in MATLAB with 4GB RAM, Intel core i5. The performance of proposed technique are compared with prior techniques to appraise the proficiency in terms of performance metrics.

\subsection{Quantitative Analysis}

In this section, two images (Figure A \& Figure B) are utilized to analyze the performance of filters.

In this study, performance evaluation is validate by calculating the SSIM, MSE and PSNR parameters. The performance evaluation is defined for two random images with different variance $(\sigma)$ of Gaussian Noise. In following tables, the performance of proposed model is measured by adding $\sigma=0.01,0.04 \& 0.1$ of Gaussian noise in original images.

Table 1: FWF filter performance at $\sigma=0.01$

\begin{tabular}{crrr}
\hline Image & \multicolumn{4}{c}{ Gaussian Noise $\sigma=0.01$} \\
\hline & SSIM & MSE & PSNR \\
& & & \\
A & $\mathbf{0 . 7 7 0 6 1}$ & 28.707764 & $\mathbf{8 7 . 5 5 9 6 3 8}$ \\
B & $\mathbf{0 . 8 0 1 7 0}$ & $\mathbf{3 0 . 7 6 7 0 7 6}$ & $\mathbf{5 4 . 4 9 7 0 2 4}$ \\
\hline
\end{tabular}
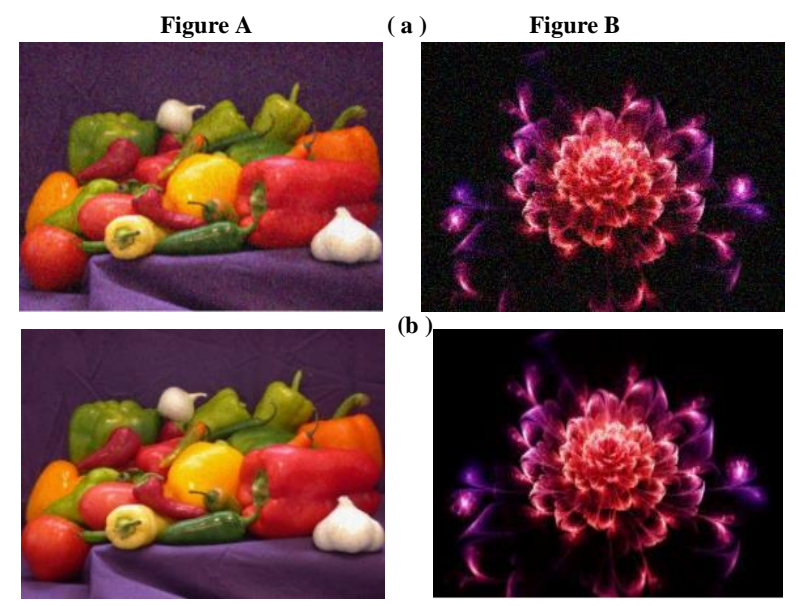

Fig. 3: (a) Images with $\sigma=0.01$ (b) De-noised images

Table 2: FWF filter performance at $\sigma=0.04$

\begin{tabular}{rrrr}
\hline Image & \multicolumn{3}{c}{ Gaussian Noise $\sigma=0.04$} \\
\hline & SSIM & MSE & PSNR \\
& & & \\
A & $\mathbf{0 . 7 8 9 0 4 3}$ & 29.755500 & $\mathbf{8 0 . 5 0 9 0 0 8}$ \\
B & $\mathbf{0 . 9 1 8 9 0 0}$ & 30.960096 & 53.998564
\end{tabular}
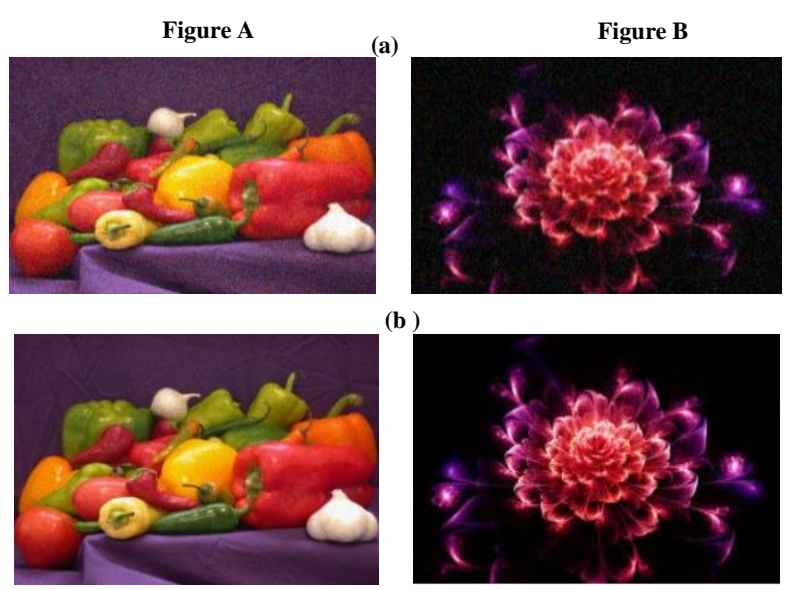

Fig. 4: (a) Images with $\sigma=0.04$ (b) De-noised images 
Table 3: FWF filter performance at $\sigma=0.1$

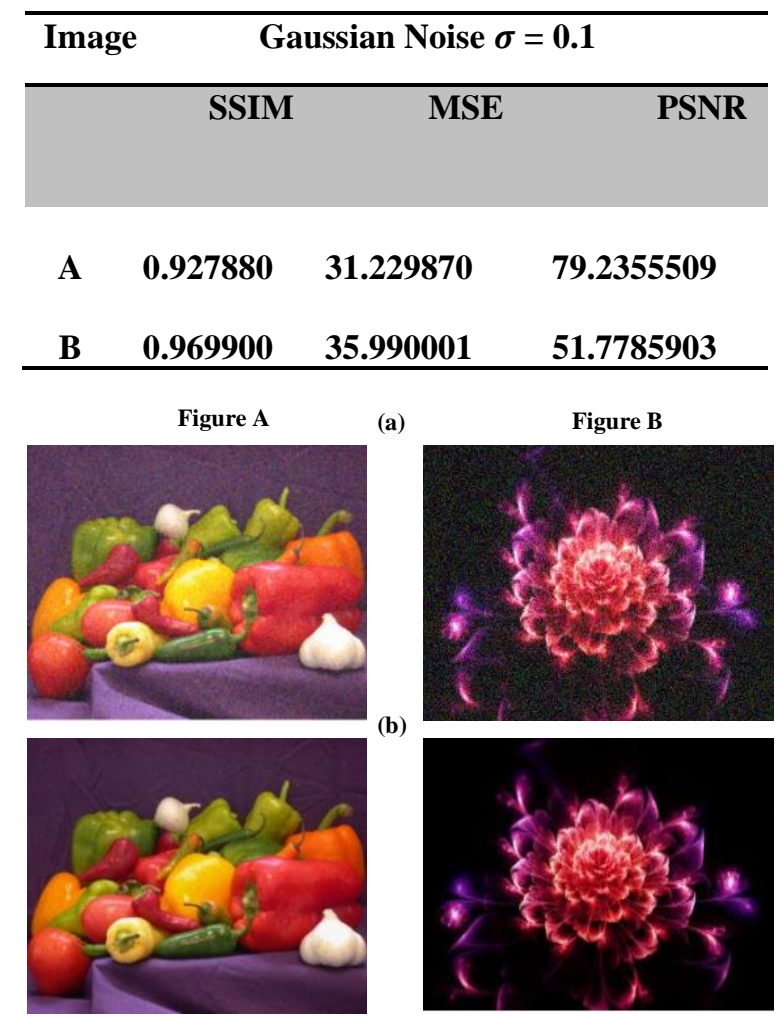

Fig. 5: (a) Images with $\sigma=0.1(\mathrm{~b})$ De-noised image

\subsection{Comparative Analysis}

Existing filter methodologies needs a lot of edge data for image de-noising, which is very limitedly available in images. In this study a FWF model proposed for distinguishing the edge information of images. In Table 4 , Table 5 and Table 6 the comparative analysis is done between the presented hybrid model and the traditional filters i.e. Weiner and Fuzzy filters. The efficiency of presented filter is verified by adding the Gaussian Noise $\sigma=0.1,0.04$.and 0.01 . The prior filters works classically in noisy pixels of images as compared to proposed filter.
Table 4: Comparative analysis of SSIM

\begin{tabular}{|c|c|c|c|c|}
\hline \multirow[t]{2}{*}{ Figure } & \multirow[t]{2}{*}{ Filter } & $\begin{array}{c}\text { GN } \\
\sigma= \\
(\mathbf{0 . 0 1})\end{array}$ & $\begin{array}{c}\text { GN } \\
\sigma= \\
(\mathbf{0 . 0 4})\end{array}$ & $\begin{array}{l}\text { GN } \\
\sigma= \\
(0.1)\end{array}$ \\
\hline & & \multicolumn{3}{|l|}{ SSIM } \\
\hline \multirow{3}{*}{$\mathbf{A}$} & Wiener & 1.27 & 1.39 & 1.45 \\
\hline & Fuzzy & 1.00 & 1.10 & 1.08 \\
\hline & Proposed & 0.77 & 0.78 & 0.92 \\
\hline \multirow{3}{*}{ B } & Wiener & 1.24 & 1.12 & 1.46 \\
\hline & Fuzzy & 1.03 & 1.01 & 1.19 \\
\hline & Proposed & 0.94 & 0.86 & 0.99 \\
\hline
\end{tabular}
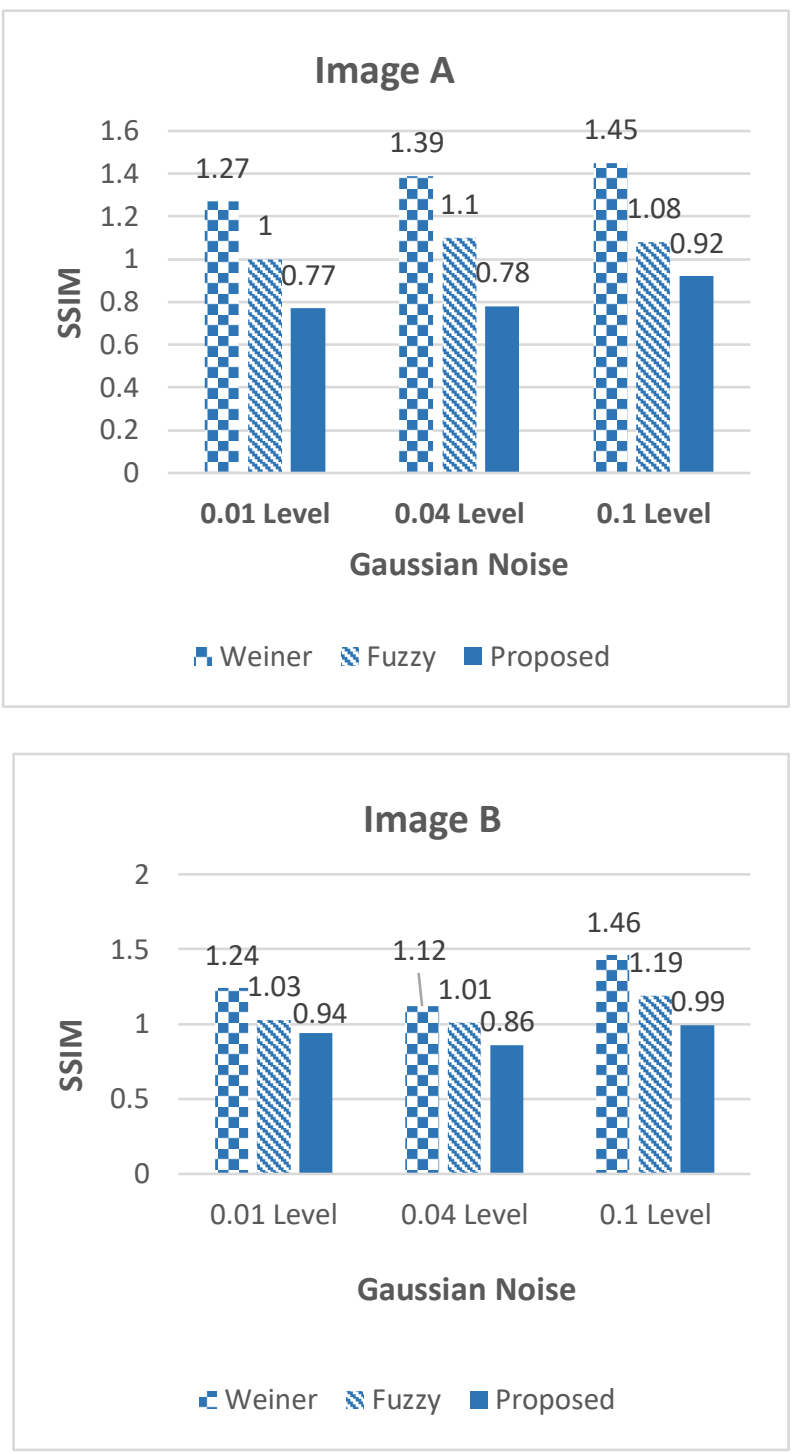

Fig. 6: Graphical comparison of SSIM 


\begin{tabular}{|c|c|c|c|c|}
\hline \multirow[t]{2}{*}{ Image } & \multirow[t]{2}{*}{ Filters } & $\begin{array}{c}\text { GN } \\
\sigma= \\
0.01\end{array}$ & $\begin{array}{c}\text { GN } \\
\sigma= \\
0.04\end{array}$ & $\begin{array}{c}\text { GN } \\
\sigma= \\
0.1\end{array}$ \\
\hline & & \multicolumn{3}{|l|}{ MSE } \\
\hline \multirow{3}{*}{$\begin{array}{l}\text { Image } \\
\text { A }\end{array}$} & Weiner & 43.22 & 46.89 & 48.98 \\
\hline & Fuzzy & 33.05 & 36.11 & 39.56 \\
\hline & $\begin{array}{l}\text { Propose } \\
\text { d }\end{array}$ & 28.70 & 29.75 & 31.22 \\
\hline \multirow{3}{*}{$\begin{array}{l}\text { Image } \\
\text { B }\end{array}$} & Weiner & 43.20 & 41.57 & 44.01 \\
\hline & Fuzzy & 37.99 & 38.09 & 40.02 \\
\hline & $\begin{array}{l}\text { Propose } \\
\text { d }\end{array}$ & 30.76 & 30.96 & 35.99 \\
\hline
\end{tabular}

Table 5: Comparative analysis of MSE

\section{Image A}

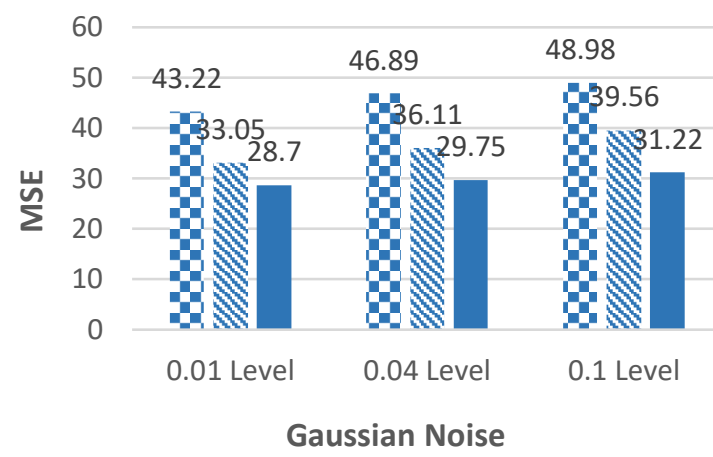

DWeiner Fuzzy $\quad$ Proposed

Image B

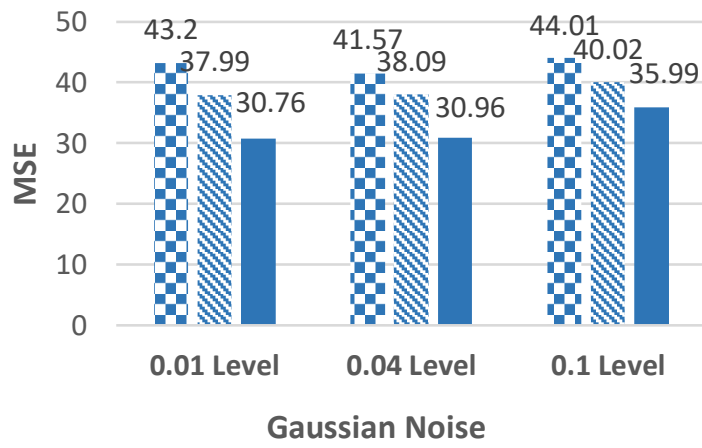

- Weiner Fuzzy $\square$ Proposed

\begin{tabular}{|c|c|c|c|c|}
\hline \multirow[t]{2}{*}{ Image } & \multirow[t]{2}{*}{ Filters } & $\begin{array}{c}\text { GN } \\
\sigma= \\
0.01\end{array}$ & $\begin{array}{c}\text { GN } \\
\sigma= \\
0.04\end{array}$ & $\begin{array}{c}\text { GN } \\
\sigma= \\
0.1\end{array}$ \\
\hline & & \multicolumn{3}{|c|}{ PSNR (dB) } \\
\hline \multirow{3}{*}{$\underset{\mathbf{A}}{\text { Image }}$} & Weiner & 60.34 & 54.12 & 48.98 \\
\hline & Fuzzy & 79.99 & 68.99 & 67.12 \\
\hline & Proposed & 87.55 & 80.50 & 79.23 \\
\hline \multirow{3}{*}{$\begin{array}{l}\text { Image } \\
\text { B }\end{array}$} & Weiner & 38.98 & 37.56 & 32.90 \\
\hline & Fuzzy & 46.78 & 43.12 & 42.09 \\
\hline & Proposed & 54.49 & 53.99 & 51.77 \\
\hline
\end{tabular}

Table 6: Comparative analysis i of PNSR

Image A

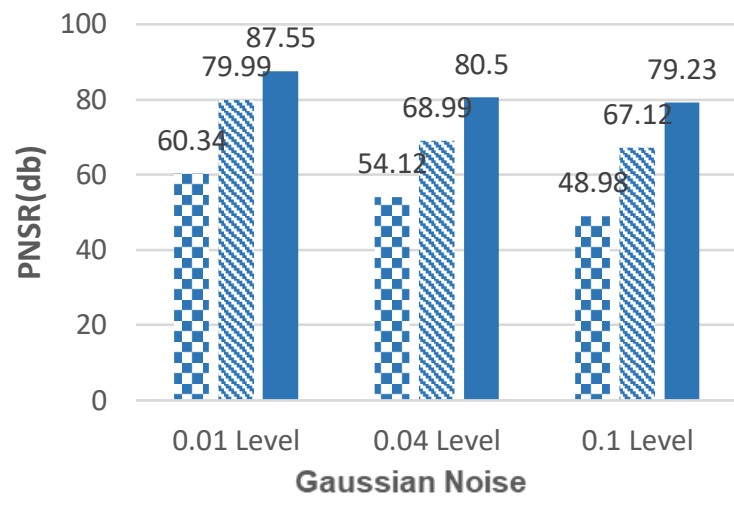

- Weiner N Fuzzy — Proposed

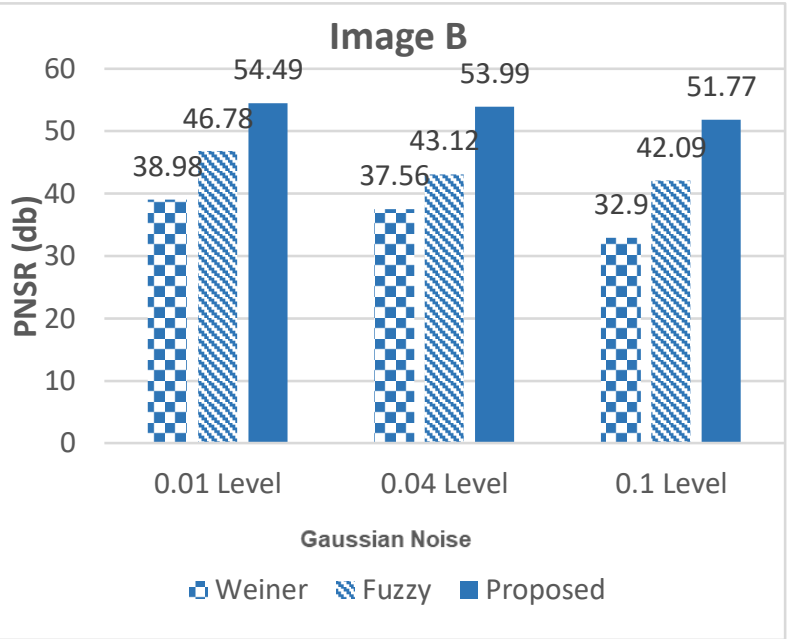

Fig. 8: Graphical comparison of PSNR

Fig. 7: Graphical comparison of MSE 


\section{SIMULATED RESULT AND DISCUSSION}

The quality of the image is degraded when noise is introduced in the image during transmission and acquisition processes. Therefore, improving the image quality is very essential in numerous applications like object detection, image retrieval, biometric verification etc. In this research, proposed model (FMF) is to reduce the noise and retain the edge information in digital images. The analysis is done by the performance evaluation parameters such as SSIM, MSE and PSNR. The proficiency of the existing filters and the proposed filter are validated under the different levels of Gaussian noise. From the simulated results, proposed model showed 0.5 to 0.8 improvement in SSIM, error rate (MSE) reduced about 10 to 12 and 15 to $20 \mathrm{~dB}$ enhancement in PSNR. The main advantage and the key benefit of this proposed (FMF) filter are more robust against Gaussian noise and efficiently preserve the edges of the digital image. From the experimental simulation, proposed filter (FMF) shows the best recovery and restoration of the noisy image which shows in figure 9. The presented hybrid filter model capable to perform intelligently for visually and subjectively.

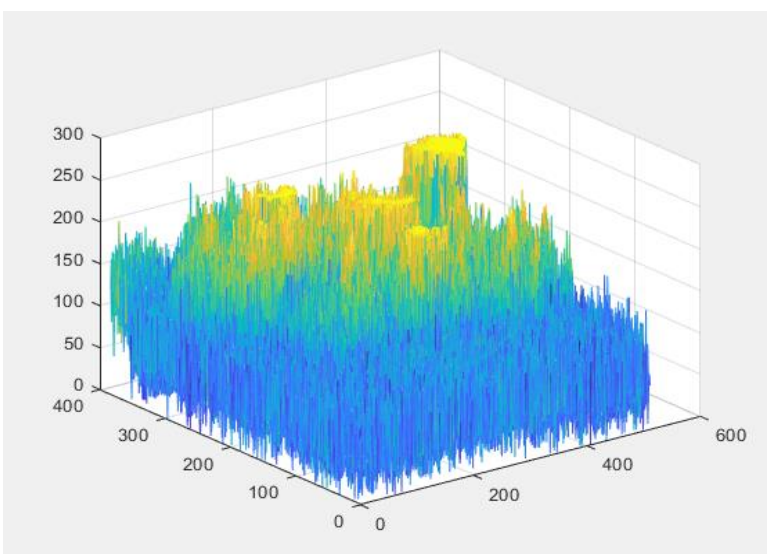

Fig. 9: GUI representation of noisy image

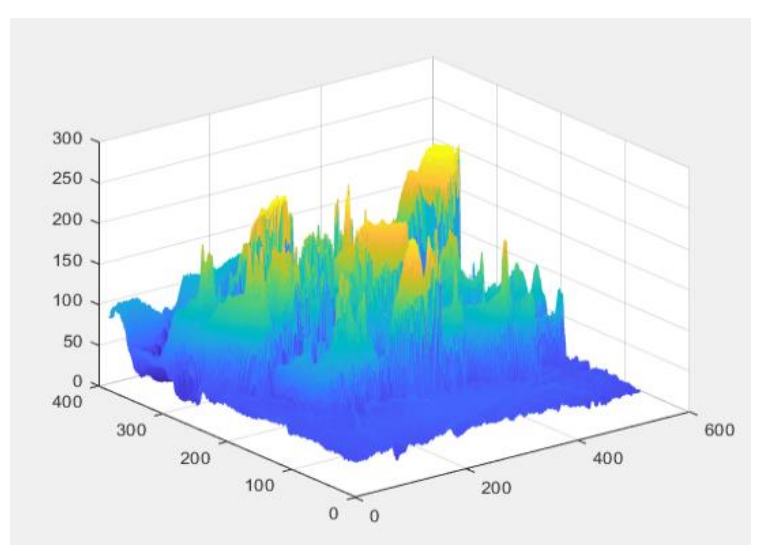

Fig. 10: GUI representation of proposed filtered image

\section{CONCLUSION}

Images are suffered with noise especially in digital image processing when the level of noise is excessively high. To address this issue, various algorithm, techniques and scheme are established for many applications like analysis of image or in the pattern recognition etc. for reducing the noise level. In this paper, hybrid model (FWF) is proposed for improving the robustness and noiselessness of the images. For checking the dynamic characteristics of proposed model, images are simulated at various Gaussian noise variance's levels. Results Comparison between the existing filters, the results shows that proposed model has improved the significant performance by means of SSIM, MSE and PSNR. The FWF hybrid model has achieved 0.5 to 0.8 SSIM, reduced MSE about 10 to $12 \%$ and 15 to $20 \mathrm{~dB}$ enhancement in PSNR.

The main key benefit of this proposed filter is that it is more vigorous against Gaussian noise and efficiently preserve the edges of the image and gives best retrieval of noisy image for compression, transmission, and recognition.

\section{REFERENCES}

[1] A. Sharma and J. Singh, "Image denoising using spatial domain filters: A quantitative study, " International Congress on Image and Signal Processing (CISP), Hangzhou, 2018, pp. 293-298

[2] Chan Rok Park, Seong-Hyeon Kang, Youngjin Lee, "Median modified wiener filter for improving the image quality of gamma camera images," Nuclear Engineering and Technology, 2020, vol 52, pp. 2328-2333

[3] T. Bhattacharya and A. Chatterjee, "Evaluating performance of some common filtering techniques for removal of Gaussian noise in images," IEEE International Conference on Power, Control, Signals and Instrumentation Engineering (ICPCSI), Chennai, 2017, pp. 1981-1984

[4] S. Khetkeeree and S. Liangrocapart, "Image Restoration Using Optimized Weiner Filtering Based on Modified Tikhonov Regularization," IEEE 4th International Conference on Signal and Image Processing (ICSIP), Wuxi, China, 2019, pp. 10151020

[5] V. V. Sazonov, M. A. Shcherbakov and V. A. Vasilyev, "Modified Wiener filter," International Conference on Biomedical Engineering and Computational Technologies (SIBIRCON), Novosibirsk, 2015, pp. 193-196

[6] Y. Zhang, J. Xiao, J. Peng, Y. Ding, J. Liu, Z. Guo, and X. Zong, "Kernel Wiener Filtering Model with Low-Rank Approximation for Image Denoising," Information Sciences, 2018, vol.462, pp.402-416 
[7] X. Zhang, "Image denoising using local Wiener filter and its method noise," Optic-International Journal for Light and Electron Optics, 2016, vol.127, pp.6821-6828

[8] M. Chowdhury, Junbin Gao and R.Islam, "Fuzzy logic based filtering for image de-noising," IEEE International Conference on Fuzzy Systems, Vancouver, 2016, pp. 2372-2376

[9] M.H. Chowdhury, M. E. Islam, N. Begum, and M.A. Bhuiyan, "Digital Image Enhancement with Fuzzy Rule-based Filtering," 10th International Conference on Computer and Information Technology, Dhaka, 2008, pp. 250-252

[10] S.Esakkirajan, T.Veerakumar, Subramanyam, C.H. Prem Chand, "Removal of high density Salt and pepper noise through modified decision based Unsymmetrical trimmed median filter," IEEE Signal Process, 2011, Lett. 18, pp. 287 290

[11] Asamoah, Dominic \& Oppong, Emmanuel \& Oppong, Stephen \& Danso, Juliana, "Measuring the Performance of Image Contrast Enhancement Technique," International Journal of Computer Applications, 2018, vol.181, pp. 6-13

[12] Al Zahir, S., \& Kashanchi, F. “A new image quality measure,” 26th IEEE Canadian Conference on Electrical and Computer Engineering (CCECE), 2013

[13] Islam, M. T., Rahman, S. M., Ahmad, M. O., \& Swamy, M. N. S.," Mixed Gaussian-impulse noise reduction from images using convolutional neural network.", Signal Processing: Image Communication, ,2018, vol. 68, pp. 26-41.

[14] Guan, Juntao, Rui Lai, Ai Xiong, Zesheng Liu, and Lin GU. "Fixed pattern noise reduction for infrared images based on cascade residual attention CNN." Neurocomputing 377, 2020,pp. 301-313.

[15] Duarte-Salazar, Carlos A., Andrés Eduardo Castro-Ospina, Miguel A. Becerra, and Edilson Delgado-Trejos. "Speckle noise reduction in ultrasound images for improving the metrological evaluation of biomedical applications: an overview." IEEE Access 8, 2020, pp. 15983-15999. 